\title{
Numerical Solution of Duffing Equation by Using an Improved Taylor Matrix Method
}

\author{
Berna Bülbül$^{1}$ and Mehmet Sezer ${ }^{2}$ \\ ${ }^{1}$ Department of Mathematics, Faculty of Science, Muğla University, 48000 Muğla, Turkey \\ ${ }^{2}$ Department of Mathematics, Faculty of Science, Celal Bayar University, 45000 Manisa, Turkey
}

Correspondence should be addressed to Mehmet Sezer; mehmet.sezer@cbu.edu.tr

Received 30 December 2012; Revised 14 May 2013; Accepted 20 May 2013

Academic Editor: Han H. Choi

Copyright ( $) 2013$ B. Bülbül and M. Sezer. This is an open access article distributed under the Creative Commons Attribution License, which permits unrestricted use, distribution, and reproduction in any medium, provided the original work is properly cited.

We have suggested a numerical approach, which is based on an improved Taylor matrix method, for solving Duffing differential equations. The method is based on the approximation by the truncated Taylor series about center zero. Duffing equation and conditions are transformed into the matrix equations, which corresponds to a system of nonlinear algebraic equations with the unknown coefficients, via collocation points. Combining these matrix equations and then solving the system yield the unknown coefficients of the solution function. Numerical examples are included to demonstrate the validity and the applicability of the technique. The results show the efficiency and the accuracy of the present work. Also, the method can be easily applied to engineering and science problems.

\section{Introduction}

Nonlinear ordinary differential equations are frequently used to model a wide class of problems in many areas of scientific fields: chemical reactions, spring-mass systems bending of beams, resistor-capacitor-inductance circuits, pendulums, the motion of a rotating mass around another body, and so forth $[1,2]$. Also, nonlinear equations which can be modeled by the oscillator equations are of crucial importance in all areas of engineering sciences [3-7]. Thus, methods of solution for these equations are of great importance to engineers and scientists. Many new techniques have appeared in the open literature such as homotopy perturbation transform method [8], variational iteration method [9], energy balance method [10], Hamiltonian approach [11], coupled homotopy-variational formulation [12], variational approach [13], and amplitude-frequency formulation [14].

In this paper, a new method is introduced for the following model of nonlinear problems:

$$
y^{\prime \prime}(x)+p y^{\prime}(x)+p_{1} y(x)+p_{2} y^{3}(x)=f(x),
$$

with the initial conditions

$$
y(0)=\alpha, \quad y^{\prime}(0)=\beta,
$$

where $p, p_{1}, p_{2}, \alpha$, and $\beta$ are real constants.

Equation (1) has been discussed in many works [15-21], for different systems arising in various scientific fields such as physics, engineering, biology, and communication theory. Recently Wang [19] presented the quasi-two-step method for the nonlinear undamped Duffing equation. Donnagáin and Rasskazov [15] studied a modification of the Duffing equation describing a periodically driven iron pendulum in nonuniform magnetic field. Feng [16] illustrated a connection between the Duffing equation and Hirota equation and obtained two periodic wave solutions in terms of elliptic functions of the Hirota equation, by using the exact solution of Duffing equation. A direct method to find the exact solution to the damped Duffing equation and traveling wave solutions to the reaction-diffusion equation was used by Feng [17]. In addition, the solution of the Duffing equation in nonlinear vibration problem by using target function method was investigated by Chen [18]. The Laplace decomposition method for numerical solution of Duffing equation has been 
introduced by Yusufoğlu [20] and Khuri [21]. On the other hand, Duffing differential equations have also been effectively dealt in many works [22-29].

The aim of this study is to get solution as truncated Taylor series centered about zero defined by

$$
y_{N}(x)=\sum_{n=0}^{N} y_{n} x^{n}, \quad y_{n}=\frac{y^{(n)}(0)}{n !}, 0 \leq x \leq b
$$

which is Taylor polynomial of degree $N$ at $x=0$, where $y_{n}, n=0,1, \ldots, N$ are unknown Taylor coefficients to be determined.

\section{Fundamental Relations}

In this section we convert the expressions defined in (1) and (2) to the matrix forms by the following procedure. Firstly, the function $y(x)$ defined by (3) can be written in the matrix form

$$
y(x)=\mathbf{X}(x) \mathbf{Y}
$$

where

$$
\mathbf{X}(x)=\left[\begin{array}{lllll}
1 & x & x^{2} & \cdots & x^{N}
\end{array}\right], \quad \mathbf{Y}=\left[\begin{array}{llll}
y_{0} & y_{1} & \cdots & y_{N}
\end{array}\right]^{T} .
$$

On the other hand, it is clearly seen that the relation between the matrix $\mathbf{X}(x)$ and its derivative $\mathbf{X}^{\prime}(x)$ is

$$
\mathbf{X}^{\prime}(x)=\mathbf{X}(x) \mathbf{B},
$$

where

$$
\mathbf{B}=\left[\begin{array}{ccccc}
0 & 1 & 0 & \cdots & 0 \\
0 & 0 & 2 & \cdots & 0 \\
\vdots & \vdots & \vdots & \ddots & \vdots \\
0 & 0 & 0 & \cdots & N \\
0 & 0 & 0 & \cdots & 0
\end{array}\right]
$$

From the matrix equations (4) and (6), it follows that

$$
\begin{gathered}
y^{\prime}(x)=\mathbf{X}^{\prime}(x) \mathbf{Y}=\mathbf{X}(x) \mathbf{B Y}, \\
y^{\prime \prime}(x)=\mathbf{X}^{\prime}(x) \mathbf{B Y}=\mathbf{X}(x) \mathbf{B}^{2} \mathbf{Y}, \\
\vdots \\
y^{(k)}(x)=\mathbf{X}^{\prime}(x) \mathbf{B}^{k-1} \mathbf{Y}=\mathbf{X}(x) \mathbf{B}^{k} \mathbf{Y}, \quad k=0,1, \ldots
\end{gathered}
$$

By using the production of two series, the matrix form of expression $y^{2}(x)$ is obtained as

$$
y^{2}(x)=\widetilde{\mathbf{X}}(x) \widetilde{\mathbf{Y}},
$$

where

$$
\begin{gathered}
\widetilde{\mathbf{X}}(x)=\left[\begin{array}{lllll}
\widetilde{\mathbf{X}}_{\mathbf{0}}(x) & \widetilde{\mathbf{X}}_{1}(x) & \cdots & \widetilde{\mathbf{X}}_{N}(x)
\end{array}\right]_{1 \times(N+1)^{2}} \\
\widetilde{\mathbf{X}}_{i}(x)=\left[\begin{array}{lllll}
x^{i} x^{0} & x^{i} x^{1} & \cdots & x^{i} x^{N}
\end{array}\right] \quad \text { or } \quad \widetilde{\mathbf{X}}_{i}(x)=x^{i} \mathbf{X}(x),
\end{gathered}
$$

$$
\begin{gathered}
\tilde{\mathbf{Y}}=\left[\begin{array}{llll}
\widetilde{\mathbf{Y}}_{0} & \tilde{\mathbf{Y}}_{1} & \cdots & \widetilde{\mathbf{Y}}_{N}
\end{array}\right]_{(N+1)^{2} \times 1}^{T}, \\
\widetilde{\mathbf{Y}}_{i}=\left[\begin{array}{llll}
y_{i} y_{0} & y_{i} y_{1} & \cdots & y_{i} y_{N}
\end{array}\right]^{T}, \quad i=0,1, \ldots, N .
\end{gathered}
$$

Similarly, the matrix representation of $y^{3}(x)$ becomes

$$
y^{3}(x)=\widetilde{\widetilde{\mathbf{X}}}(x) \widetilde{\widetilde{\mathbf{Y}}},
$$

where

$$
\begin{aligned}
& \widetilde{\widetilde{\mathbf{X}}}=\left[\begin{array}{llll}
\widetilde{\widetilde{\mathbf{X}}}_{\mathbf{0}}(x) & \widetilde{\widetilde{\mathbf{X}}}_{\mathbf{1}}(x) & \cdots & \widetilde{\widetilde{\mathbf{X}}}_{N}(x)
\end{array}\right]_{1 \times(N+1)^{3}}, \\
& \widetilde{\widetilde{\mathbf{X}}}_{i}(x)=x^{i} \widetilde{\mathbf{X}}(x), \\
& \tilde{\widetilde{\mathbf{Y}}}=\left[\begin{array}{llll}
\tilde{\widetilde{\mathbf{Y}}}_{0} & \tilde{\widetilde{\mathbf{Y}}}_{1} & \ldots & \tilde{\widetilde{\mathbf{Y}}}_{N}
\end{array}\right]_{(N+1)^{3} \times 1}^{T}, \quad \tilde{\widetilde{\mathbf{Y}}}_{i}=y_{i} \tilde{\mathbf{Y}}(x), \\
& i=0,1, \ldots, N \text {. }
\end{aligned}
$$

Finally, by substituting the matrix forms (4)-(11) into (1) we have the fundamental matrix equation

$$
\mathbf{X}(x) \mathbf{B}^{2} \mathbf{Y}+p \mathbf{X}(x) \mathbf{B Y}+p_{1} \mathbf{X}(x) \mathbf{Y}+p_{2} \widetilde{\widetilde{\mathbf{X}}}(x) \widetilde{\widetilde{\mathbf{Y}}}=f(x)
$$

\section{Method of the Solution}

To obtain the Taylor polynomial solution of (1) in the form (3) we firstly compute the Taylor coefficients by means of the collocation points defined by

$$
x_{i}=\frac{b}{N} i, \quad i=0,1, \ldots, N .
$$

By substituting the collocation points into matrix equation (13) we obtain the system of matrix equations

$\mathbf{X}\left(x_{i}\right) \mathbf{B}^{2} \mathbf{Y}+p \mathbf{X}\left(x_{i}\right) \mathbf{B} \mathbf{Y}+p_{1} \mathbf{X}\left(x_{i}\right) \mathbf{Y}+p_{2} \widetilde{\widetilde{\mathbf{X}}}\left(x_{i}\right) \tilde{\widetilde{\mathbf{Y}}}=f\left(x_{i}\right)$, $i=0,1, \ldots, N$,

or compact notation

$$
\mathbf{X B}^{2} \mathbf{Y}+p \mathbf{X B Y}+p_{1} \mathbf{X Y}+p_{2} \widetilde{\widetilde{\mathbf{X}}} \widetilde{\mathbf{Y}}=\mathbf{F} .
$$

Briefly, we can write the matrix equation

$$
\mathbf{W Y}+\mathbf{V} \widetilde{\widetilde{Y}}=\mathbf{F} \text {, }
$$

which corresponds to a system of $(N+1)$ nonlinear algebraic equations with the unknown Taylor coefficients $y_{n}, n=$ $0,1, \ldots, N$. The matrices in (17) are as follows:

$$
\mathbf{W}=\left[w_{i, j}\right]=\mathbf{X B}^{2}+p \mathbf{X B}+p_{1} \mathbf{X},
$$

$$
i, j=0,1, \ldots, N
$$

$$
\mathbf{V}=\left[v_{m, n}\right]=p_{2} \widetilde{\widetilde{\mathbf{X}}}
$$

$m=0,1, \ldots, N, n=0,1, \ldots,\left((N+1)^{3}-1\right)$, 
where

$$
\mathbf{X}=\left[\begin{array}{c}
\mathbf{X}\left(x_{0}\right) \\
\mathbf{X}\left(x_{1}\right) \\
\vdots \\
\mathbf{X}\left(x_{N}\right)
\end{array}\right], \quad \widetilde{\widetilde{\mathbf{X}}}=\left[\begin{array}{c}
\widetilde{\mathbf{X}}\left(x_{0}\right) \\
\widetilde{\mathbf{X}}\left(x_{1}\right) \\
\vdots \\
\widetilde{\mathbf{X}}\left(x_{N}\right)
\end{array}\right], \quad \mathbf{F}=\left[\begin{array}{c}
f\left(x_{0}\right) \\
f\left(x_{1}\right) \\
\vdots \\
f\left(x_{N}\right)
\end{array}\right]
$$

The augmented matrix for (17) becomes, clearly,

$$
[\mathbf{W} ; \mathbf{V}: \mathbf{F}]=\left[\begin{array}{ccccccccccc}
w_{0,0} & w_{0,1} & \cdots & w_{0, N} & ; & v_{0,0} & v_{0,1} & \cdots & v_{0,(N+1)^{3}-1} & ; & f\left(x_{0}\right) \\
w_{1,0} & w_{1,1} & \cdots & w_{1, N} & ; & v_{1,0} & v_{1,1} & \cdots & v_{1,(N+1)^{3}-1} & f\left(x_{0}\right) \\
\vdots & \vdots & \vdots & \vdots & \vdots & \vdots & \vdots & \vdots & \vdots & \vdots & \vdots \\
w_{N, 0} & w_{N, 1} & \cdots & w_{N, N} & ; & v_{N, 0} & v_{N, 1} & \cdots & v_{N,(N+1)^{3}-1} & ; & f\left(x_{N}\right)
\end{array}\right]
$$

Next, by means of relations (4) and (8), we can obtain the corresponding matrix forms for the initial conditions (2) as

$$
\begin{aligned}
& \mathbf{X}(0) \mathbf{Y}=\alpha \quad \text { or } \quad\left[\begin{array}{llllllllll}
1 & 0 & \cdots & 0 & ; & 0 & 0 & \cdots & 0 & ;
\end{array}\right] \text {, } \\
& \mathbf{X}(0) \mathbf{B Y}=\beta \quad \text { or } \quad\left[\begin{array}{lllllllllll}
0 & 1 & \cdots & 0 & ; & 0 & 0 & \cdots & 0 & ;
\end{array}\right] \text {. }
\end{aligned}
$$

Consequently, to obtain the solution of (1) under the conditions (2), by replacing the row matrices (21) by the last two rows of the augmented matrix (20), we have the required augmented matrix

$$
[\widetilde{\mathbf{W}} ; \quad \widetilde{\mathbf{V}}: \quad \widetilde{\mathbf{F}}]=\left[\begin{array}{ccccccccccc}
w_{0,0} & w_{0,1} & \cdots & w_{0, N} & ; & v_{0,0} & v_{0,1} & \cdots & v_{0,(N+1)^{3}-1} & \vdots & f\left(x_{0}\right) \\
w_{1,0} & w_{1,1} & \cdots & w_{1, N} & ; & v_{1,0} & v_{1,1} & \cdots & v_{1,(N+1)^{3}-1} & \vdots & f\left(x_{0}\right) \\
\vdots & \vdots & \vdots & \vdots & \vdots & \vdots & \vdots & \cdots & \vdots & \vdots & \vdots \\
w_{(N-2), 0} & w_{(N-2), 1} & \cdots & w_{(N-2), N} & ; & v_{(N-2), 0} & v_{(N-2), 1} & \cdots & v_{(N-2),(N+1)^{3}-1} & \vdots & f\left(x_{N-2}\right) \\
1 & 0 & \cdots & 0 & ; & 0 & 0 & \cdots & 0 & \vdots & \alpha \\
0 & 1 & \cdots & 0 & ; & 0 & 0 & \cdots & 0 & \vdots & \beta
\end{array}\right]
$$

or

$$
\widetilde{\mathbf{W}} \mathbf{Y}+\widetilde{\mathbf{V}} \widetilde{\tilde{\mathbf{Y}}}=\widetilde{\mathbf{F}} .
$$

By solving the matrix equation (22), the unknown Taylor coefficients $y_{n}$ are determined. Thus we get the Taylor polynomial solution

$$
y_{N}(x)=\sum_{n=0}^{N} y_{n} x^{n}
$$

\section{Error Bound and Accuracy of the Solution}

We can easily check the accuracy of the solution $y_{N}(x)$ as follows. Since the Taylor polynomial (3) is an approximate solution of (1), then the solution $y_{N}(x)$ is substituted in (1), and the resulting equation must be satisfied approximately; that is, for $x=x_{i} \in[a, b], i=0,1,2, \ldots$,

$$
\begin{gathered}
E_{N}\left(x_{i}\right)=\mid \\
y_{N}^{\prime \prime}\left(x_{i}\right)+p y_{N}^{\prime}\left(x_{i}\right)+p_{1} y_{N}\left(x_{i}\right) \\
+p_{2} y_{N}^{3}\left(x_{i}\right)-f\left(x_{i}\right) \mid \cong 0
\end{gathered}
$$

or $E_{N}\left(x_{i}\right) \leq 10^{-k_{i}}$ ( $k_{i}$ positive integer).
If $\max \left(10^{k_{i}}\right)=10^{-k}$ ( $k$ positive integer) is prescribed, then the truncation limit $N$ is increased until the difference $E_{N}\left(x_{i}\right)$ at each of the points becomes smaller than the prescribed $10^{-k}$. On the other hand, the error can be estimated by means of the function

$$
\begin{aligned}
E_{N}(x)= & y_{N}^{\prime \prime}(x)+p y_{N}^{\prime}(x) \\
& +p_{1} y_{N}(x)+p_{2} y_{N}^{3}(x)-f(x) .
\end{aligned}
$$

If $E_{N}(x) \rightarrow 0$, when $N$ is sufficiently large enough, then the error decreases.

Also if we know the exact solution of problem, we can find error bound of method.

Theorem 1 (Lagrange error bound). Let $f$ be function such that it and all of its derivatives are continuous, and $f_{n}(x)$ is the nth Taylor polynomial for $f(x)$ centered $x=a$; then the error is bounded by

$$
\left|f(x)-f_{n}(x)\right|=\left|E_{n}(x)\right| \leq \frac{M}{(n+1) !}|x-a|^{n+1},
$$

where $M=\max \left|f^{(n+1)}(\xi)\right|, \xi \in(a, x)$. 


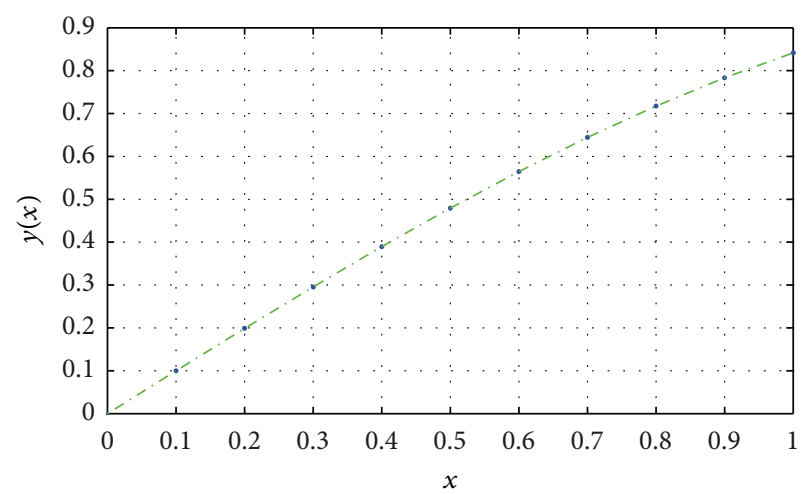

- Exact solution

- - Numerical solution

(a)

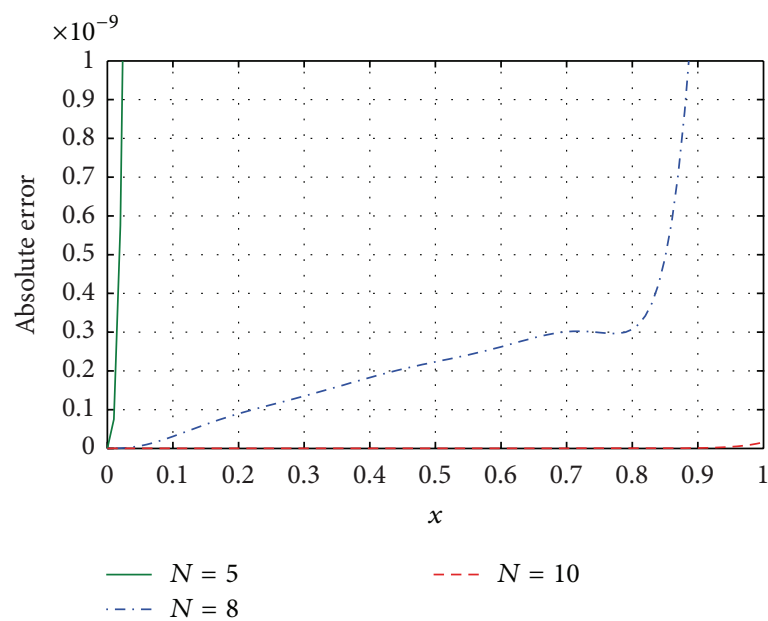

(b)

Figure 1: (a) Comparison of the approximate solution with exact solution for $N=10$. (b) Comparison of absolute errors for $N=5,8$, and 10 .

TABLE 1: Comparison of the absolute errors of Example 1.

\begin{tabular}{lcccc}
\hline$x_{r}$ & Exact & \multicolumn{2}{c}{ Error analysis $\left(\left|y-y_{N}\right|\right)$} & $N=8$ \\
\hline 0 & 0 & 0 & 0 & 0 \\
0.1 & 0.9983341665 & $4.62 E-8$ & $3.08 E-11$ & $4.33 E-14$ \\
0.2 & 0.1986693308 & $6.12 E-7$ & $8.72 E-11$ & $1.03 E-13$ \\
0.3 & 0.2955202067 & $4.28 E-7$ & $1.45 E-11$ & $1.66 E-13$ \\
0.4 & 0.3894183423 & $2.29 E-7$ & $1.82 E-10$ & $2.21 E-13$ \\
0.5 & 0.4794255386 & $4.23 E-7$ & $1.65 E-10$ & $2.71 E-13$ \\
0.6 & 0.5646424734 & $4.03 E-7$ & $2.62 E-10$ & $3.15 E-13$ \\
0.7 & 0.6442176872 & $3.32 E-7$ & $3.01 E-12$ & $2.29 E-13$ \\
0.8 & 0.7173560909 & $5.66 E-7$ & $3.07 E-10$ & $3.85 E-13$ \\
0.9 & 0.7833260096 & $8.87 E-6$ & $5.68 E-9$ & $9.73 E-13$ \\
1 & 0.841470984 & $1.43 E-5$ & $1.22 E-8$ & $1.57 E-11$ \\
\hline
\end{tabular}

If $y_{N}$ is approximate solution of the matrix method and $f_{N}(x)$ is the $N$ th Taylor polynomial for $f(x)$, we can write $\left|f_{N}-y_{N}\right|=\varepsilon$, and absolute error function of the method is $\left|f-y_{N}\right|=e_{N}$. To obtain error bound by Theorem 1 we have

$$
\begin{aligned}
\frac{M}{(N+1) !}|x-a|^{N+1} & \geq\left|f_{N}-f\right|=\left|f_{N}-f+y_{N}-y_{N}\right| \\
& \geq|| f_{N}-y_{N}|-| f-y_{N}||, \\
\frac{M}{(N+1) !}|x-a|^{N+1} & \geq\left|\varepsilon-e_{N}\right| \Longrightarrow \varepsilon-\frac{M}{(N+1) !}|x-a|^{N+1} \\
& \leq e_{N} \leq \varepsilon+\frac{M}{(N+1) !}|x-a|^{N+1} .
\end{aligned}
$$

Therefore we can write

$$
e_{N} \leq \max _{x \in[a, b]} \varepsilon+\frac{M}{(N+1) !}|x-a|^{N+1},
$$

which gives us error bound.

\section{Numerical Examples}

The method of this study is useful in finding the solutions of Duffing equations in terms of Taylor polynomials. We illustrate it by the following examples. Numerical computations have been done using Maple 9.

Example 1. Consider the Duffing equation in the following type [20]:

$$
y^{\prime \prime}(x)+3 y(x)-2 y^{3}(x)=\cos (x) \sin (2 x),
$$

with the initial conditions

$$
y(0)=0, \quad y^{\prime}(0)=1 .
$$

We assume that the problem has a Taylor polynomial solution in the form

$$
y_{N}(x)=\sum_{n=0}^{N} y_{n} x^{n}, \quad \text { where } N=5,8, \text { and } 10 .
$$


TABLE 2: Numerical results of Example 2.

\begin{tabular}{|c|c|c|c|c|}
\hline \multirow{2}{*}{$x_{r}$} & \multicolumn{4}{|c|}{ Error analysis $\left(E_{N}\left(x_{r}\right)\right)$} \\
\hline & $N=5$ & $N=10$ & $N=15$ & $N=20$ \\
\hline 0 & 0 & 0 & $1.70 E-8$ & 0 \\
\hline 0.1 & $4.62 E-2$ & 0 & 0 & 0 \\
\hline 0.2 & $5.57 E-24$ & 0 & $1.05 E-9$ & $2.23 E-25$ \\
\hline 0.3 & $2.24 E-3$ & 0 & 0 & $2.22 E-25$ \\
\hline 0.4 & 0 & $3.22 E-24$ & $2.91 E-11$ & $9.80 E-25$ \\
\hline 0.5 & $6.00 E-25$ & 0 & 0 & $1.00 E-24$ \\
\hline 0.6 & 0 & $2.51 E-24$ & $1.62 E-11$ & $1.70 E-24$ \\
\hline 0.7 & $3.63 E-2$ & $4.31 E-24$ & 0 & $1.31 E-24$ \\
\hline 0.8 & $1.13 E-1$ & $1.87 E-4$ & $1.80 E-24$ & $2.10 E-24$ \\
\hline 0.9 & $1.46 E-1$ & $8.28 E-3$ & $1.32 E-7$ & $8.50 E-24$ \\
\hline 1 & $5.93 E-1$ & $1.32 E-2$ & $4.47 E-5$ & $3.68 E-24$ \\
\hline
\end{tabular}

The solutions obtained for $N=5,8$, and 10 are compared with the exact solution $y(x)=\sin (x)$ [20] (see Table 1 and Figure 1). We calculate the following Lagrange error bound for different values of $N$, respectively, $M_{5}=0.161 \times 10^{-2}$ which is 2 decimal places accuracy, $M_{8}=0.352 \times 10^{-5}$ which is 5 decimal places accuracy, and $M_{10}=0.248 \times 10^{-7}$ which is 7 decimal places accuracy.

Example 2. Consider the Duffing equation [20]

$$
y^{\prime \prime}(x)+0.4 y^{\prime}(x)+1.1 y(x)+y^{3}(x)=0,
$$

with initial conditions

$$
y(0)=0.3, \quad y^{\prime}(0)=-2.3 .
$$

Applying the present method, we have the following fundamental matrix equation:

$$
\left\{\mathbf{X B}^{2}+0.4 \mathbf{X B}+1.1 \mathbf{X}\right\} \mathbf{Y}+\widetilde{\widetilde{\mathbf{X}}} \widetilde{\mathbf{Y}}=\mathbf{F}, \quad \mathbf{F}=\left[\begin{array}{llll}
0 & 0 & \cdots & 0
\end{array}\right]_{(N+1) \times 1}^{T} .
$$

The solution of this nonlinear system is obtained for $N=$ $5,10,15$, and 20. For numerical results, see Tables 2, 3 and Figure 2.

Example 3. In this last example, the method is illustrated by considering the damped Duffing equation [21]

$$
\begin{aligned}
& y^{\prime \prime}(x)+k y^{\prime}=-y^{3}(x), \\
& y(0)=\alpha, \quad y^{\prime}(0)=\beta .
\end{aligned}
$$

Consider the case $\alpha=\beta=k=1$.

We have similar results for Example 3 as in Example 2. Table 4 shows the error values $E_{N}\left(x_{r}\right)$ for $N=5,8,10$, and 20.

\section{Conclusion}

In this paper, a very simple but effective Taylor matrix method was proposed for the numerical solution of the nonlinear
TABLE 3: Comparison of numerical solutions.

\begin{tabular}{lcc}
\hline$x_{r}$ & $\tilde{y}(x)$ (Laplace method) & $y_{N}(x)$ (present method) \\
\hline 0 & 0.3 & 0.3 \\
0.1 & 0.0835845348 & 0.08358453484 \\
0.2 & -0.105091915 & -0.1050919158 \\
0.3 & -0.266020303 & -0.2660203036 \\
0.4 & -0.399977948 & -0.3999779482 \\
0.5 & -0.508314810 & -0.5083148108 \\
0.6 & -0.592890689 & -0.5928906891 \\
0.7 & -0.656065502 & -0.6560655028 \\
0.8 & -0.700676939 & -0.7006769399 \\
0.9 & -0.729970850 & -0.7299708508 \\
1 & -0.747476077 & -0.7474760775 \\
\hline
\end{tabular}

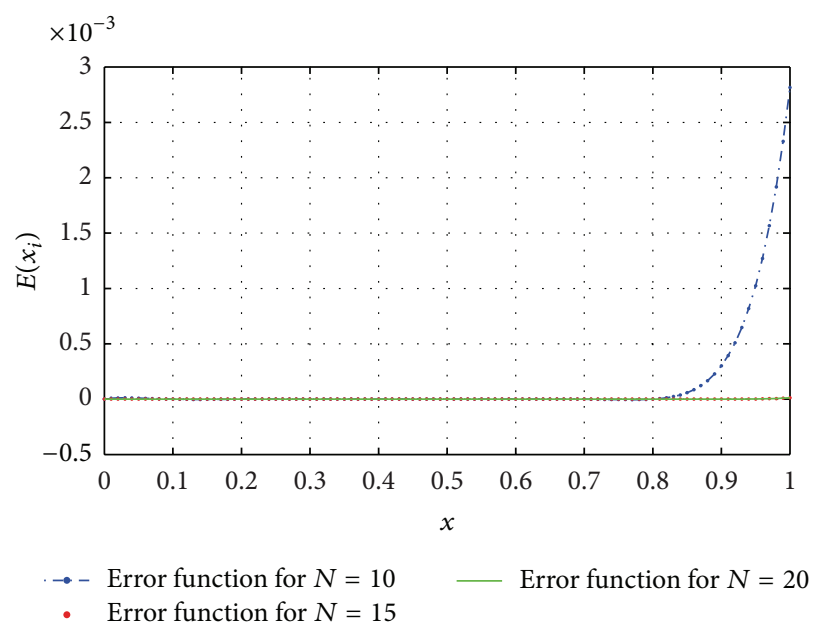

FIGURE 2: Error functions for $N=10,15$, and 20 of Example 2.

Duffing equation. One of the advantages of this method that is the solution is expressed as a truncated Taylor series, then $y(x)$ can be easily evaluated for arbitrary values of $x$ by using the computer program without any computational effort. From the given illustrative examples, it can be seen 
TABLE 4: Comparison of the absolute errors of Example 3.

\begin{tabular}{|c|c|c|c|c|}
\hline \multirow{2}{*}{$x_{r}$} & \multicolumn{4}{|c|}{ Error analysis $\left(E_{N}\left(x_{r}\right)\right)$} \\
\hline & $N=5$ & $N=8$ & $N=10$ & $N=20$ \\
\hline 0 & 0 & 0 & 0 & 0 \\
\hline 0.1 & $4.62 E-3$ & $5.08 E-6$ & 0 & 0 \\
\hline 0.2 & 0 & $6.72 E-6$ & 0 & 0 \\
\hline 0.3 & $4.54 E-3$ & $1.45 E-6$ & 0 & 0 \\
\hline 0.4 & 0 & $3.82 E-6$ & 0 & 0 \\
\hline 0.5 & $7.85 E-3$ & 0 & 0 & 0 \\
\hline 0.6 & 0 & $2.62 E-5$ & 0 & 0 \\
\hline 0.7 & $5.42 E-2$ & $3.01 E-5$ & 0 & 0 \\
\hline 0.8 & $1.94 E-1$ & $8.07 E-4$ & 0 & 0 \\
\hline 0.9 & $0.46 E-1$ & $5.68 E-3$ & 0 & 0 \\
\hline 1 & $0.93 E-1$ & $1.22 E-2$ & $4.57 E-8$ & $3.32 E-29$ \\
\hline
\end{tabular}

that the Taylor series approach can obtain very accurate and satisfactory results. However, the main point is that the polynomial expansion is highly ill-posed when $x$ is larger than 1 . This method can be improved with new strategies to solve the other nonlinear equations.

\section{References}

[1] G. F. Corliss, Guarented Error Bounds for Ordinary Differential Equations, in Theory and Numeric of Ordinary and Partial Equations, Oxford University press, Oxford, UK, 1995.

[2] H. Bulut and D. J. Evans, "On the solution of the Riccati equation by the decomposition method," International Journal of Computer Mathematics, vol. 79, no. 1, pp. 103-109, 2002.

[3] Y. Khan, M. Mada, A. Yildirim, M. A. Abdou, and N. Faraz, "A new approach to van der Pol's oscillator problem," Zeitschrift für Naturforschung A, vol. 66, pp. 620-624, 2011.

[4] Y. Khan, H. Vázquez-Leal, and N. Faraz, "An efficient new iterative method for oscillator differential equation," Scientia Iranica, vol. 19, pp. 1473-1477, 2012.

[5] Y. Khan, "Mehdi Akbarzade Dynamic analysis of non-linear oscillator equation arising in double-sided driven clamped microbeam-based electromechanical resonator," Zeitschrift für Naturforschung A, vol. 67, pp. 435-440, 2012.

[6] Y. Khan, M. Akbarzade, and A. Kargar, "Coupling of homotopy and variational approach for conservative oscillator with strong odd-nonlinearity," Scientia Iranica, vol. 19, pp. 417-422, 2012.

[7] M. Akbarzade and Y. Khan, "Dynamic model of large amplitude non-linear oscillations arising in the structural engineering: analytical solutions," Mathematical and Computer Modelling, vol. 55, no. 3-4, pp. 480-489, 2012.

[8] Y. Khan and Q. Wu, "Homotopy perturbation transform method for nonlinear equations using He's polynomials," Computers \& Mathematics with Applications, vol. 61, no. 8, pp. 19631967, 2011.

[9] J. H. He, G. C. Wu, and F. Austin, "The variational iteration method which should be followed," Nonlinear Science Letters A, vol. 1, pp. 1-30, 2010.

[10] N. Jamshidi and D. D. Ganji, "Application of energy balance method and variational iteration method to an oscillation of a mass attached to a stretched elastic wire," Current Applied Physics, vol. 10, pp. 484-486, 2010.
[11] J.-H. He, "Hamiltonian approach to nonlinear oscillators," Physics Letters A, vol. 374, no. 23, pp. 2312-2314, 2010.

[12] M. Akbarzade and J. Langari, "Determination of natural frequencies by coupled method of homotopy perturbation and variational method for strongly nonlinear oscillators," Journal of Mathematical Physics, vol. 52, no. 2, Article ID 023518, 10 pages, 2011.

[13] J.-H. He, "Variational approach for nonlinear oscillators," Chaos, Solitons \& Fractals, vol. 34, no. 5, pp. 1430-1439, 2007.

[14] D. D. Ganji and M. Akbarzade, "Approximate analytical solutions to non-linear oscillators using He's amplitude-frequency formulation," International Journal of Mathematical Analysis, vol. 32, no. 4, pp. 1591-1597, 2010.

[15] M. O. Donnagáin and O. Rasskazov, "Numerical modelling of an iron pendulum in a magnetic field," Physica B, vol. 372, no. 1-2, pp. 37-39, 2006.

[16] Z. Feng, "Duffing's equation and its applications to the Hirota equation," Physics Letters A, vol. 317, no. 1-2, pp. 115-119, 2003.

[17] Z. Feng, "Monotonous property of non-oscillations of the damped Duffing's equation," Chaos, Solitons and Fractals, vol. 28, no. 2, pp. 463-471, 2006.

[18] Y. Z. Chen, "Solution of the Duffing equation by using target function method," Journal of Sound and Vibration, vol. 256, no. 3, pp. 573-578, 2002.

[19] Z. Wang, "P-stable linear symmetric multistep methods for periodic initial-value problems," Computer Physics Communications, vol. 171, no. 3, pp. 162-174, 2005.

[20] E. Yusufoğlu, "Numerical solution of Duffing equation by the Laplace decomposition algorithm," Applied Mathematics and Computation, vol. 177, no. 2, pp. 572-580, 2006.

[21] S. A. Khuri, "A Laplace decomposition algorithm applied to a class of nonlinear differential equations," Journal of Applied Mathematics, vol. 1, no. 4, pp. 141-155, 2001.

[22] C. Pezeshki and E. H. Dowell, "An examination of initial condition maps for the sinusoidally excited buckled beam modeled by the Duffing's equation," Journal of Sound and Vibration, vol. 117, no. 2, pp. 219-232, 1987.

[23] S. A. Khuri and S. Xie, "On the numerical verification of the asymptotic expansion of Duffing's equation," International Journal of Computer Mathematics, vol. 372, no. 3, pp. 25-30, 1999.

[24] Y. H. Ku and X. Sun, "CHAOS and limit cycle in Duffing's equation," Journal of the Franklin Institute, vol. 327, no. 2, pp. 165-195, 1990.

[25] F. Battelli and K. J. Palmer, "Chaos in the Duffing equation," Journal of Differential Equations, vol. 101, no. 2, pp. 276-301, 1993.

[26] A. Loria, E. Panteley, and H. Nijmeijer, "Control of the chaotic Duffing equation with uncertainty in all parameters," IEEE Transactions on Circuits and Systems. I. Fundamental Theory and Applications, vol. 45, no. 12, pp. 1252-1255, 1998.

[27] H. Nijmeijer and H. Berghuis, "On Lyapunov control of the Duffing equation," IEEE Transactions on Circuits and Systems. I. Fundamental Theory and Applications, vol. 42, no. 8, pp. 473477, 1995.

[28] J. G. Byatt-Smith, "Regular and chaotic solutions of Duffing's equation for large forcing," IMA Journal of Applied Mathematics, vol. 37, no. 2, pp. 113-145, 1986.

[29] P. Habets and G. Metzen, "Existence of periodic solutions of Duffing equations," Journal of Differential Equations, vol. 78, no. 1, pp. 1-32, 1989. 


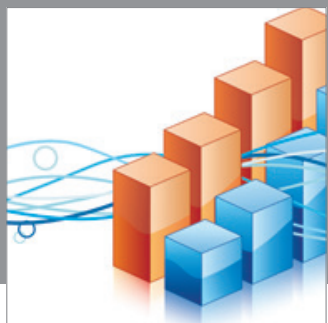

Advances in

Operations Research

mansans

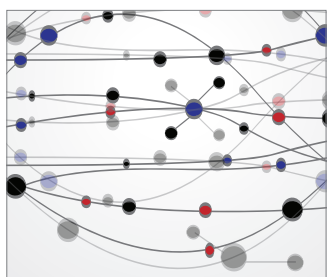

The Scientific World Journal
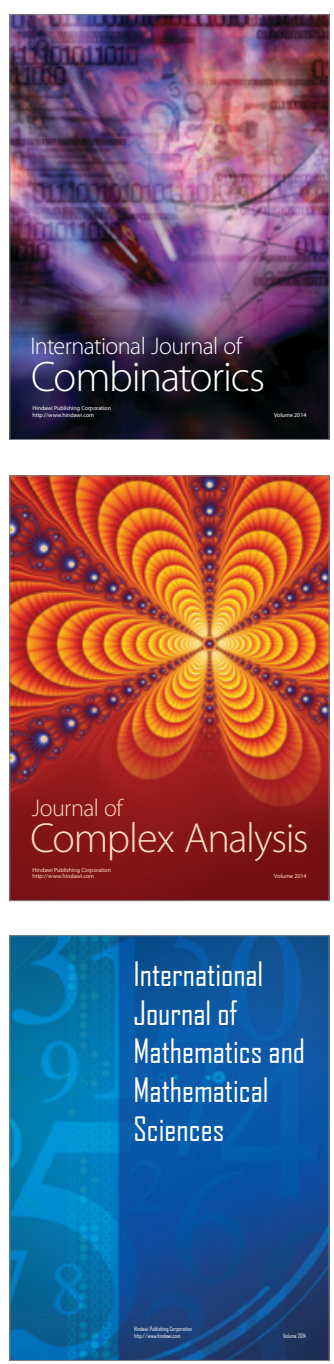
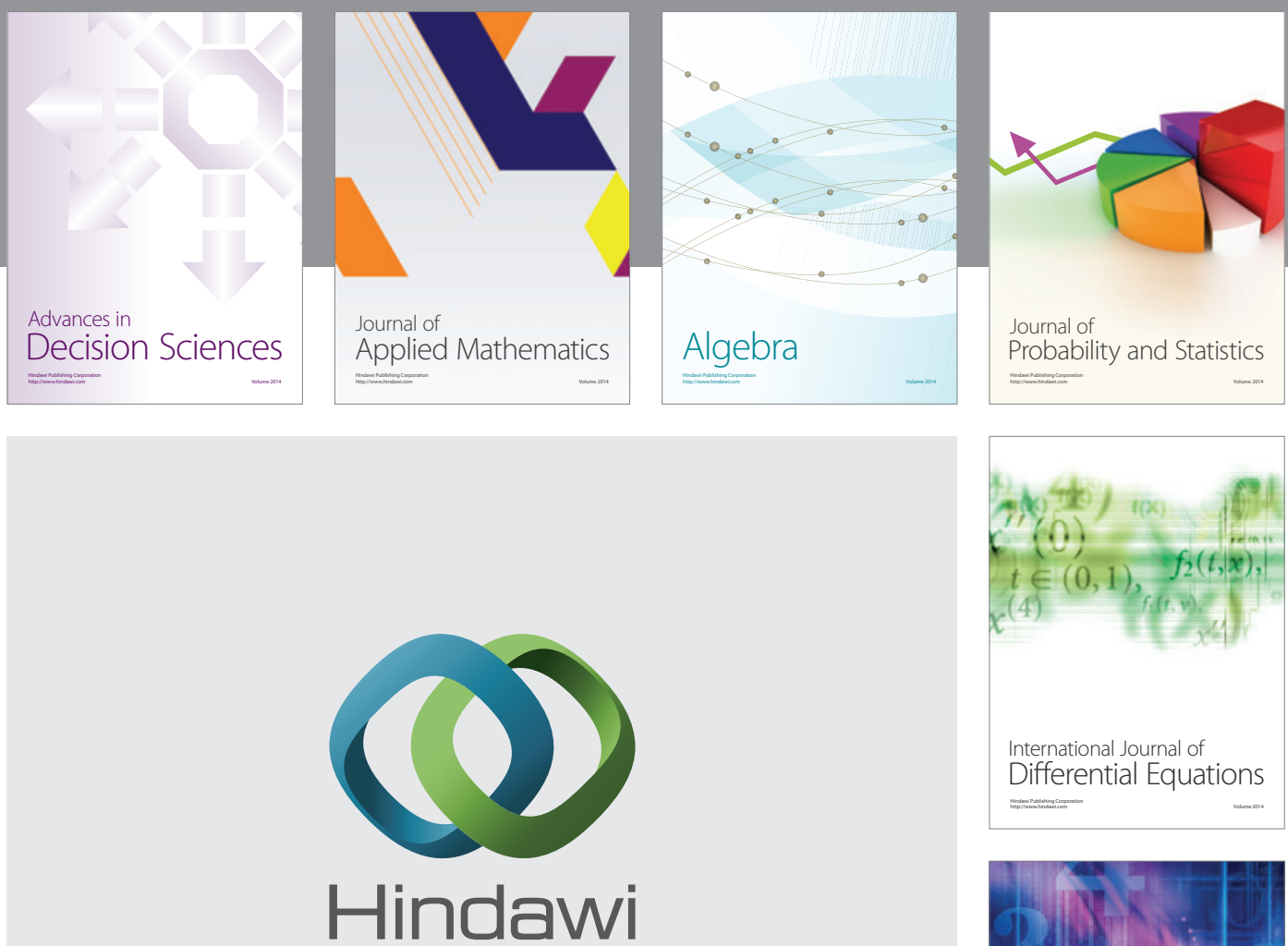

Submit your manuscripts at http://www.hindawi.com
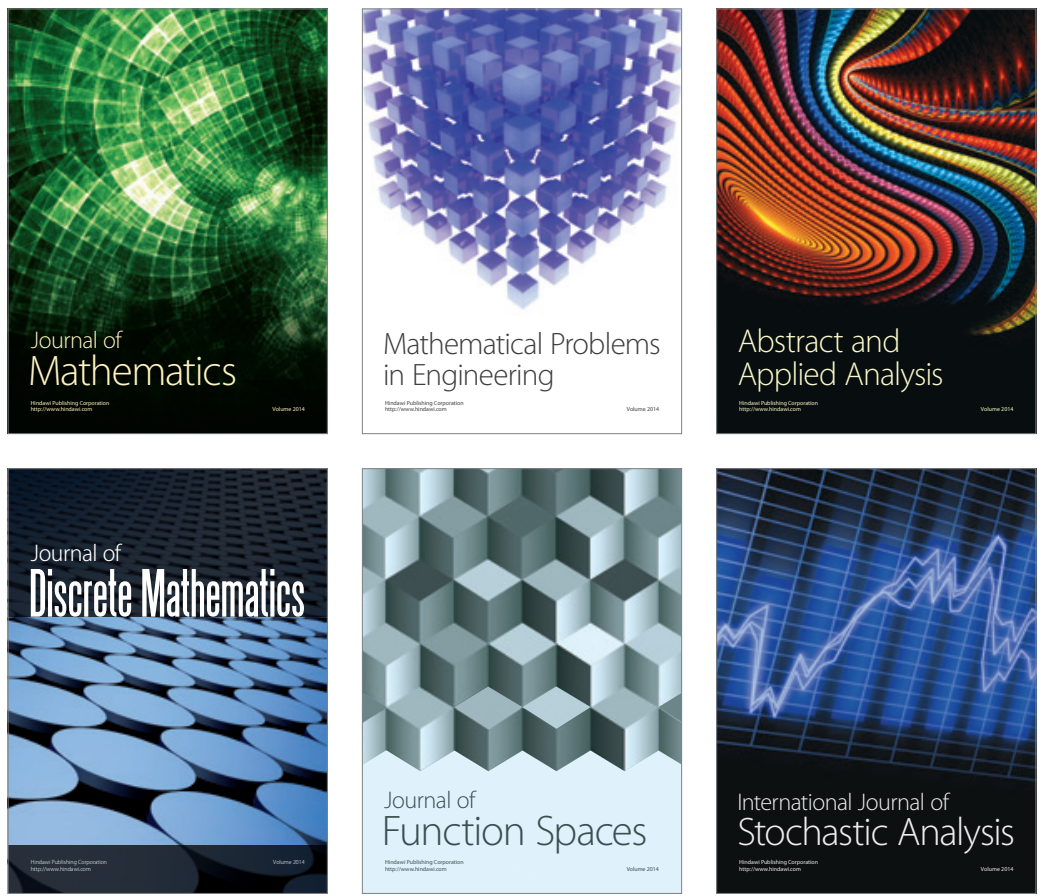

Journal of

Function Spaces

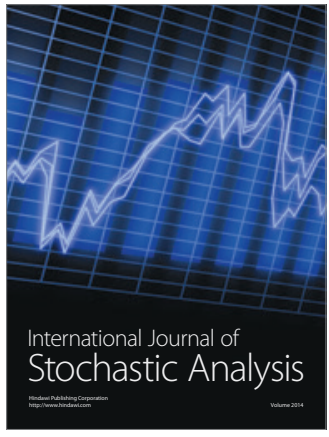

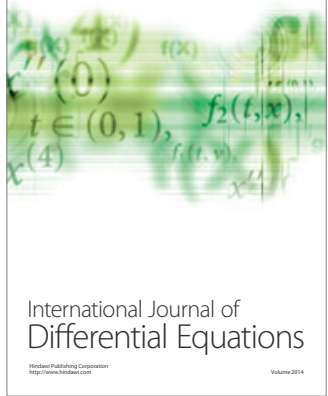
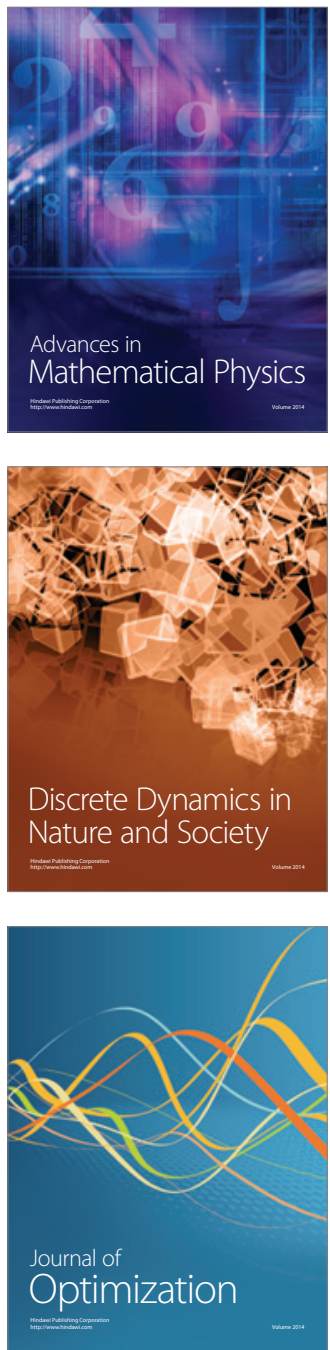